\title{
Coloring Intersection Graphs of Arc-Connected Sets in the Plane
}

\author{
Michał Lasoń • Piotr Micek • Arkadiusz Pawlik • \\ Bartosz Walczak
}

Received: 7 February 2014 / Revised: 21 May 2014 / Accepted: 5 July 2014 /

Published online: 13 August 2014

(C) The Author(s) 2014. This article is published with open access at Springerlink.com

\begin{abstract}
A family of sets in the plane is simple if the intersection of any subfamily is arc-connected, and it is pierced by a line $L$ if the intersection of any member with $L$ is a nonempty segment. It is proved that the intersection graphs of simple families of compact arc-connected sets in the plane pierced by a common line have chromatic number bounded by a function of their clique number.
\end{abstract}

Keywords Geometric intersection graphs $\cdot$ Chromatic number $\cdot \chi$-boundedness

\section{Introduction}

A proper coloring of a graph is an assignment of colors to the vertices of the graph such that no two adjacent ones are assigned the same color. The minimum number of colors sufficient to color a graph $G$ properly is called the chromatic number of $G$ and

M. Lasoń · P. Micek · A. Pawlik · B. Walczak

Theoretical Computer Science Department, Faculty of Mathematics and Computer Science,

Jagiellonian University, Kraków, Poland

e-mail:walczak@tcs.uj.edu.pl

M. Lasoń

e-mail: michalason@gmail.com

P. Micek

e-mail: micek@tcs.uj.edu.pl

A. Pawlik

e-mail: pawlik@tcs.uj.edu.pl

M. Lasoń

Institute of Mathematics of the Polish Academy of Sciences, Warsaw, Poland

M. Lasoń · B. Walczak

École Polytechnique Fédérale de Lausanne, Lausanne, Switzerland 
denoted by $\chi(G)$. The maximum size of a clique (a set of pairwise adjacent vertices) in a graph $G$ is called the clique number of $G$ and denoted by $\omega(G)$. It is clear that $\chi(G) \geqslant \omega(G)$.

The chromatic and clique numbers of a graph can be arbitrarily far apart. There are various constructions of graphs that are triangle-free (have clique number 2) and still have arbitrarily large chromatic number. The first one was given in 1949 by Zykov [16], and the one perhaps best known is due to Mycielski [11]. However, these classical constructions require a lot of freedom in connecting vertices by edges, and many important classes of graphs derived from specific (e.g. geometric) representations have chromatic number bounded in terms of the clique number. A class of graphs is called $\chi$-bounded if there is a function $f: \mathbb{N} \rightarrow \mathbb{N}$ such that $\chi(G) \leqslant f(\omega(G))$ holds for any graph $G$ from the class.

In this paper, we focus on the relation between the chromatic number and the clique number for geometric intersection graphs. The intersection graph of a family of sets $\mathcal{F}$ is the graph with vertex set $\mathcal{F}$ and edge set consisting of pairs of intersecting elements of $\mathcal{F}$. We consider finite families $\mathcal{F}$ of arc-connected compact sets in the plane which are simple in the sense that the intersection of any subfamily of $\mathcal{F}$ is also arc-connected. We usually identify the family $\mathcal{F}$ with its intersection graph and use such terms as chromatic number, clique number or $\chi$-boundedness referring directly to $\mathcal{F}$.

In the one-dimensional case of subsets of $\mathbb{R}$, the only arc-connected compact sets are closed intervals. They define the class of interval graphs, which have chromatic number equal to their clique number. The study of the chromatic number of intersection graphs of geometric objects in higher dimensions was initiated in the seminal paper of Asplund and Grünbaum [1], where they proved that the families of axis-aligned rectangles in $\mathbb{R}^{2}$ are $\chi$-bounded. On the other hand, Burling [2] showed that intersection graphs of axis-aligned boxes in $\mathbb{R}^{3}$ with clique number 2 can have arbitrarily large chromatic number.

Since then, a lot of research focused on proving $\chi$-boundedness of the families of geometric objects in the plane with various restrictions on the kind of objects considered, their positions, or the way they can intersect. Gyárfás [5,6] proved that the families of chords of a circle are $\chi$-bounded. This was generalized by Kostochka and Kratochvíl [8] to the families of convex polygons inscribed in a circle. Kim et al. [7] showed that the families of homothetic (uniformly scaled) copies of a fixed convex compact set in the plane are $\chi$-bounded. Fox and Pach [3] showed that the intersection graphs of any arc-connected compact sets in the plane that do not contain a fixed bipartite subgraph $H$ have chromatic number bounded by a function of $H$. This easily implies that the families of pseudodiscs, that is, closed disc homeomorphs in the plane the boundaries of any two of which cross at most twice, are $\chi$-bounded. Note that families of pseudodiscs are simple. The above-mentioned results of [7] and [3] are actually stronger - they state that the number of edges of the intersection graph of a respective family $\mathcal{F}$ is bounded by $f(\omega(\mathcal{F}))|\mathcal{F}|$ for some function $f$.

A family of sets $\mathcal{F}$ is pierced by a line $L$ if the intersection of any member of $\mathcal{F}$ with $L$ is a nonempty segment. McGuinness [9] proved that the families of L-shapes (shapes consisting of a horizontal and a vertical segments of arbitrary lengths, forming the letter ' $L$ ') pierced by a fixed vertical line are $\chi$-bounded. Later [10], he showed that the triangle-free simple families of compact arc-connected sets in the plane pierced 
by a common line have bounded chromatic number. Suk [15] proved $\chi$-boundedness of the simple families of $x$-monotone curves intersecting a fixed vertical line. In this paper, we generalize the results of McGuinness, allowing any bound on the clique number, and of Suk, removing the $x$-monotonicity condition.

Theorem 1 The class of simple families of compact arc-connected sets in the plane pierced by a common line is $\chi$-bounded.

By contrast, Pawlik et al. [12,13] proved that there are intersection graphs of straight-line segments (or geometric sets of many other kinds) with clique number 2 and arbitrarily large chromatic number. This justifies the assumption of Theorem 1 that the sets are pierced by a common line. The best known upper bound on the chromatic number of simple families of curves in the plane with clique number $\omega$ is $\left(\frac{\log n}{\log \omega}\right)^{O(\log \omega)}$ due to Fox and Pach [4].

The bound on the chromatic number following from our proof of Theorem 1 is double exponential in terms of the clique number.

The ultimate goal of this quest is to understand the border line between the classes of graphs (and classes of geometric objects) that are $\chi$-bounded and those that are not. In a preliminary version of this paper, we proposed the following two problems.

Problem 1 Are the families (not necessarily simple) of $x$-monotone curves in the plane pierced by a common vertical line $\chi$-bounded?

Problem 2 Are the families of curves in the plane pierced by a common line $\chi$-bounded?

Rok and Walczak [14] proved recently that the answers to both these questions are positive. However, the bound on the chromatic number in terms of the clique number resulting from their proof is enormous (greater than an exponential tower), which is much worse than the double exponential bound of Theorem 1.

\section{Topological Preliminaries}

All the geometric sets that considered in this paper are assumed to be subsets of the Euclidean plane $\mathbb{R}^{2}$ or, further in the paper, subsets of the closed upper halfplane $\mathbb{R} \times[0,+\infty)$. An arc between points $x, y \in \mathbb{R}^{2}$ is the image of a continuous injective $\operatorname{map} \phi:[0,1] \rightarrow \mathbb{R}^{2}$ such that $\phi(0)=x$ and $\phi(1)=y$. A set $X \subset \mathbb{R}^{2}$ is arc-connected if any two points of $X$ are connected by an arc in $X$. The union of two arc-connected sets that have non-empty intersection is itself arc-connected. More generally, if $\mathcal{X}$ is a family of arc-connected sets whose intersection graph is connected, then $\bigcup \mathcal{X}$ is arc-connected. For a set $X \subset \mathbb{R}^{2}$, the relation $\left\{(x, y) \in X^{2}: X\right.$ contains an arc between $x$ and $y$ \} is an equivalence, whose equivalence classes are the arc-connected components of $X$. Every arc-connected component of an open set is itself an open set.

All families of sets that we consider are finite. A family $\mathcal{F}$ of sets in $\mathbb{R}^{2}$ is simple if the intersection of any subfamily of $\mathcal{F}$ is arc-connected (possibly empty). A set $X$ is simple with respect to a family $\mathcal{Y}$ if $\{X\} \cup \mathcal{Y}$ is simple. 
Lemma 2 Let $X$ be a compact arc-connected set and $\mathcal{Y}$ be a family of compact arcconnected sets such that $X$ is simple with respect to $\mathcal{Y}$ and the intersections of the members of $\mathcal{Y}$ with $X$ are pairwise disjoint. Between any points $x_{1}, x_{2} \in X$, there is an arc $A \subset X$ that is simple with respect to $\mathcal{Y}$.

Proof Let $\mathcal{Y}=\left\{Y_{1}, \ldots, Y_{n}\right\}$. For $i \in\{0, \ldots, n\}$, we construct an arc $A_{i} \subset X$ between $x_{1}$ and $x_{2}$ that is simple with respect to $\left\{Y_{1}, \ldots, Y_{i}\right\}$. As $X$ is arc-connected, we pick $A_{0}$ to be any arc between $x_{1}$ and $x_{2}$ within $X$. We construct $A_{i}$ from $A_{i-1}$ as follows. If $A_{i-1} \cap Y_{i}=\emptyset$, then we take $A_{i}=A_{i-1}$. Otherwise, let $y_{1}$ and $y_{2}$ be respectively the first and the last points on $A_{i-1}$ that belong to $Y_{i}$ (which exist as $A_{i} \cap Y_{i}$ is non-empty and compact). To obtain $A_{i}$, replace the part of $A_{i-1}$ between $y_{1}$ and $y_{2}$ by any arc between $y_{1}$ and $y_{2}$ in $X \cap Y_{i}$ (which exists because $X \cap Y_{i}$ is arc-connected). Clearly, $A_{i}$ is simple with respect to $Y_{i}$. Since $X \cap Y_{i}$ is disjoint from each of $Y_{1}, \ldots, Y_{i-1}, A_{i}$ remains simple with respect to $Y_{1}, \ldots, Y_{i-1}$.

A Jordan curve is the image of a continuous map $\phi:[0,1] \rightarrow \mathbb{R}^{2}$ such that $\phi(0)=$ $\phi(1)$ and $\phi$ is injective on $[0,1)$. The famous Jordan curve theorem states that if $C \subset \mathbb{R}^{2}$ is a Jordan curve, then $\mathbb{R}^{2} \backslash C$ has exactly two arc-connected components, one bounded and one unbounded. An extension of this, called Jordan-Schönflies theorem, adds that there is a homeomorphism of $\mathbb{R}^{2}$ that maps $C$ to a unit circle, the bounded arc-connected component of $\mathbb{R}^{2} \backslash C$ to the interior of this circle, and the unbounded arc-connected component of $\mathbb{R}^{2} \backslash C$ to the exterior of the circle.

We will use a special case of the Jordan curve theorem for arcs in the closed upper halfplane $\mathbb{R} \times[0,+\infty)$. Namely, if $x$ and $y$ are two points on the horizontal axis $\mathbb{R} \times\{0\}$ and $A$ is an arc between $x$ and $y$ such that $A \backslash\{x, y\} \subset \mathbb{R} \times(0,+\infty)$, then the set $(\mathbb{R} \times[0,+\infty)) \backslash A$ has exactly two arc-connected components, one bounded and one unbounded. This in particular implies that for any four points $x_{1}, x_{2}, y_{1}$ and $y_{2}$ in this order on the horizontal axis, every arc in $\mathbb{R} \times[0,+\infty)$ between $x_{1}$ and $y_{1}$ intersects every arc in $\mathbb{R} \times[0,+\infty)$ between $x_{2}$ and $y_{2}$.

\section{Grounded Families}

In Theorem 1, a family $\mathcal{F}$ compact arc-connected sets in the plane is assumed to be pierced by a common line. We assume without loss of generality that this piercing line is the horizontal axis $\mathbb{R} \times\{0\}$ and call it the baseline. The base of a set $X$, denoted by base $(X)$, is the intersection of $X$ with the baseline.

We fix a positive integer $k$ and assume $\omega(\mathcal{F}) \leqslant k$. The intersection graph of the bases of the members of $\mathcal{F}$ is an interval graph, so it can be properly colored with $k$ colors. To find a proper coloring of $\mathcal{F}$ with a number of colors bounded in terms of $k$, we can restrict our attention to one color class in the coloring of this interval graph. Therefore, without loss of generality, we assume that no two members of $\mathcal{F}$ intersect on the baseline and show that $\mathcal{F}$ can be colored properly with a bounded number of colors. Moreover, it is clear that the families $\mathcal{F}^{+}=\{X \cap(\mathbb{R} \times[0,+\infty)): X \in \mathcal{F}\}$ and $\mathcal{F}^{-}=\{X \cap(\mathbb{R} \times(-\infty, 0]): X \in \mathcal{F}\}$ are simple. It suffices to obtain proper colorings $\phi^{+}$and $\phi^{-}$of $\mathcal{F}^{+}$and $\mathcal{F}^{-}$, respectively, with bounded numbers of colors, since then $\mathcal{F}$ may be colored by pairs $\left(\phi^{+}, \phi^{-}\right)$. We only focus on coloring $\mathcal{F}^{+}$, as $\mathcal{F}^{-}$can be 


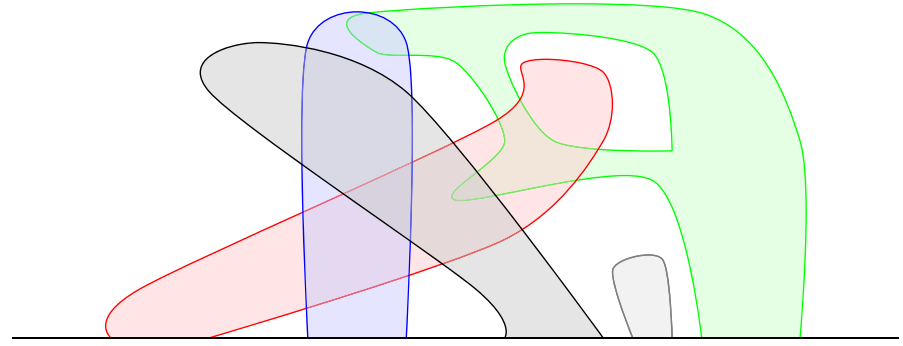

Fig. 1 A grounded family of sets

handled by symmetry. To simplify notation, we rename $\mathcal{F}^{+}$to $\mathcal{F}$. Therefore, each set $X \in \mathcal{F}$ is assumed to satisfy the following:

- $X \subset \mathbb{R} \times[0,+\infty)$,

- $X \cap \mathbb{R} \times\{0\}$ is a non-empty interval,

- $X$ is compact and arc-connected,

and $\mathcal{F}$ is assumed to be simple. Any set that satisfies the conditions above is called grounded, and any simple family of grounded sets with pairwise disjoint bases is also called grounded (see Fig. 1).

All the geometric sets that we consider from now on are contained in $\mathbb{R} \times[0,+\infty)$. To prove Theorem 1, it suffices to show the following.

Proposition 3 For $k \geqslant 1$, there is $\xi_{k}$ such that $\chi(\mathcal{F}) \leqslant \xi_{k}$ holds for any grounded family $\mathcal{F}$ with $\omega(\mathcal{F}) \leqslant k$.

The case $k=1$ is trivial, and the case $k=2$ with some additional assumptions meant to avoid topological pathologies was settled by McGuinness [10].

We write $X \prec Y$ if $\operatorname{base}(X)$ is entirely to the left of $\operatorname{base}(Y)$. The relation $\prec$ is a total order on a grounded family and naturally extends to its subfamilies (or any other families of grounded sets with pairwise disjoint bases): for example, $X \prec \mathcal{Y}$ denotes that $X \prec Y$ for any $Y \in \mathcal{Y}$. For grounded sets $X_{1}$ and $X_{2}$ such that $X_{1} \prec X_{2}$, we define $\mathcal{F}\left(X_{1}, X_{2}\right)=\left\{Y \in \mathcal{F}: X_{1} \prec Y \prec X_{2}\right\}$. For a grounded set $X$, we define $\mathcal{F}(-\infty, X)=\{Y \in \mathcal{F}: Y \prec X\}$ and $\mathcal{F}(X,+\infty)=\{Y \in \mathcal{F}: X \prec Y\}$.

The proof of Proposition 3 heavily depends on two decomposition lemmas, which given a grounded family with large chromatic number find its subfamily with large chromatic number and some special properties. The first one is a reformulation of Lemma 2.1 in [9].

Lemma 4 Let $\mathcal{F}$ be a grounded family with $\chi(\mathcal{F})>2 a(b+1)$, where $a, b \geqslant 0$. There is a subfamily $\mathcal{H}$ of $\mathcal{F}$ that satisfies $\chi(\mathcal{H})>$ a and $\chi\left(\mathcal{F}\left(H_{1}, H_{2}\right)\right)>$ b for any intersecting $H_{1}, H_{2} \in \mathcal{H}$.

Proof We partition $\mathcal{F}$ into subfamilies $\mathcal{F}_{0} \prec \cdots \prec \mathcal{F}_{n}$ so that $\chi\left(\mathcal{F}_{i}\right)=b+1$ for $0 \leqslant i<n$. This can be done by adding sets to $\mathcal{F}_{0}$ in the increasing $\prec$-order until we get $\chi\left(\mathcal{F}_{0}\right)=b+1$, then following the same procedure with the remaining sets to form $\mathcal{F}_{1}$, and so on. Let $\mathcal{F}^{0}=\bigcup_{i} \mathcal{F}_{2 i}$ and $\mathcal{F}^{1}=\bigcup_{i} \mathcal{F}_{2 i+1}$. Since $\chi\left(\mathcal{F}^{0} \cup \mathcal{F}^{1}\right)>2 a(b+1)$, 


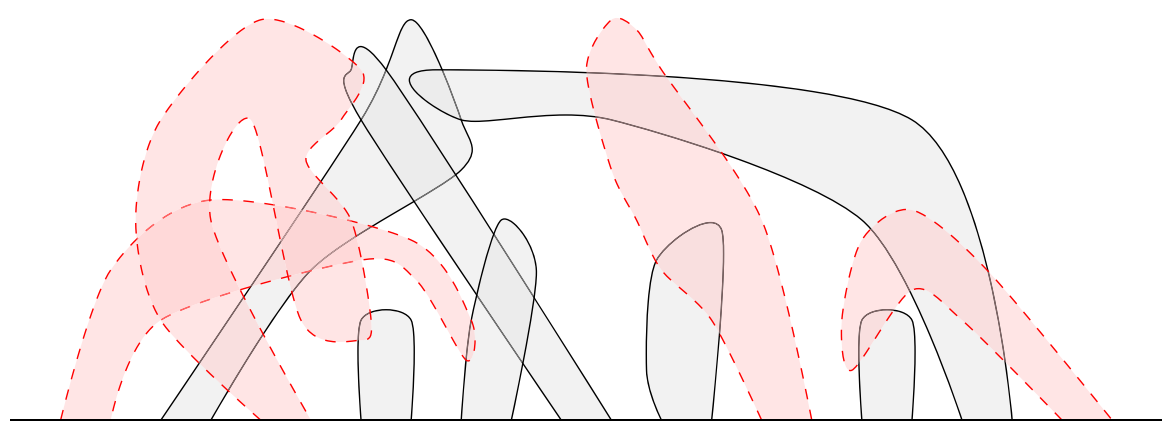

Fig. 2 An externally supported family of sets

we have $\chi\left(\mathcal{F}^{k}\right)>a(b+1)$ for $k=0$ or $k=1$. We now color each $\mathcal{F}_{2 i+k}$ properly using the same set of $b+1$ colors. This coloring induces a partitioning of the entire $\mathcal{F}^{k}$ into subfamilies $\mathcal{H}_{0}, \ldots, \mathcal{H}_{b}$ such that for $0 \leqslant i \leqslant n, 0 \leqslant j \leqslant b$ the family $\mathcal{F}_{i} \cap \mathcal{H}_{j}$ is independent. We set $\mathcal{H}=\mathcal{H}_{j}$, where $\mathcal{H}_{j}$ has the maximum chromatic number among $\mathcal{H}_{0}, \ldots, \mathcal{H}_{b}$. Since $\chi\left(\mathcal{F}^{k}\right)>a(b+1)$, we have $\chi(\mathcal{H})>a$. It remains to show that $\chi\left(\mathcal{F}\left(H_{1}, H_{2}\right)\right)>b$ for $H_{1}, H_{2} \in \mathcal{H}$ with $H_{1} \cap H_{2} \neq \emptyset$. Indeed, such sets $H_{1}$ and $H_{2}$ must lie in different families $\mathcal{F}_{2 i_{1}+k}$ and $\mathcal{F}_{2 i_{2}+k}$, respectively, so $\chi\left(\mathcal{F}\left(H_{1}, H_{2}\right)\right) \geqslant \chi\left(\mathcal{F}_{2 i_{1}+k+1}\right)=b+1>b$, as required.

For a set $X$, we define $\operatorname{ext}(X)$ to be the only unbounded arc-connected component of $(\mathbb{R} \times[0,+\infty)) \backslash X$. For a grounded family $\mathcal{F}$, we $\operatorname{define} \operatorname{ext}(\mathcal{F})=\operatorname{ext}(\bigcup \mathcal{F})$. A subfamily $\mathcal{G}$ of a grounded family $\mathcal{F}$ is externally supported in $\mathcal{F}$ if for any $X \in \mathcal{G}$ there exists $Y \in \mathcal{F}$ such that $Y \cap X \neq \emptyset$ and $Y \cap \operatorname{ext}(\mathcal{G}) \neq \varnothing$ (see Fig. 2). The idea behind the following lemma is due to Gyárfás [5] and was subsequently used in $[9,10,15]$.

Lemma 5 Let $\mathcal{F}$ be a grounded family with $\chi(\mathcal{F})>2 a$, where $a \geqslant 1$. There is $a$ subfamily $\mathcal{G}$ of $\mathcal{F}$ that is externally supported in $\mathcal{F}$ and satisfies $\chi(\mathcal{G})>a$.

Proof For convenience, we restrict $\mathcal{F}$ to its connected component with maximum chromatic number. Let $X_{0}$ be the $\prec$-least member of $\mathcal{F}$. For $i \geqslant 0$, let $\mathcal{F}_{i}$ be the family of members of $\mathcal{F}$ that are at distance $i$ from $X_{0}$ in the intersection graph of $\mathcal{F}$. It follows that $\mathcal{F}_{0}=\left\{X_{0}\right\}$ and, for $|i-j|>1$, each member of $\mathcal{F}_{i}$ is disjoint from each member of $\mathcal{F}_{j}$. Clearly, $\chi\left(\bigcup_{i} \mathcal{F}_{2 i}\right)>a$ or $\chi\left(\bigcup_{i} \mathcal{F}_{2 i+1}\right)>a$, and therefore there is $d \geqslant 1$ with $\chi\left(\mathcal{F}_{d}\right)>a$. We claim that $\mathcal{F}_{d}$ is externally supported in $\mathcal{F}$. Fix $X_{d} \in \mathcal{F}_{d}$, and let $X_{0}, \ldots, X_{d}$ be a shortest path from $X_{0}$ to $X_{d}$ in the intersection graph of $\mathcal{F}$. Since $X_{0} \cap \operatorname{ext}\left(\mathcal{F}_{d}\right) \neq \emptyset$ and $X_{0}, \ldots, X_{d-2}$ are disjoint from $\bigcup \mathcal{F}_{d}$, we have $X_{0}, \ldots, X_{d-2} \subset \operatorname{ext}\left(\mathcal{F}_{d}\right)$. Thus $X_{d-1} \cap \operatorname{ext}\left(\mathcal{F}_{d}\right) \neq \emptyset$ and $X_{d-1} \cap X_{d} \neq \emptyset$.

\section{Cliques and Brackets}

Let $\mathcal{F}$ be a grounded family with $\omega(\mathcal{F}) \leqslant k$. A $k$-clique in $\mathcal{F}$ is a family of $k$ pairwise intersecting members of $\mathcal{F}$. For a $k$-clique $\mathcal{K}$, we denote by $\operatorname{int}(\mathcal{K})$ the only arcconnected component of $(\mathbb{R} \times[0,+\infty)) \backslash \cup \mathcal{K}$ containing the part of the baseline 


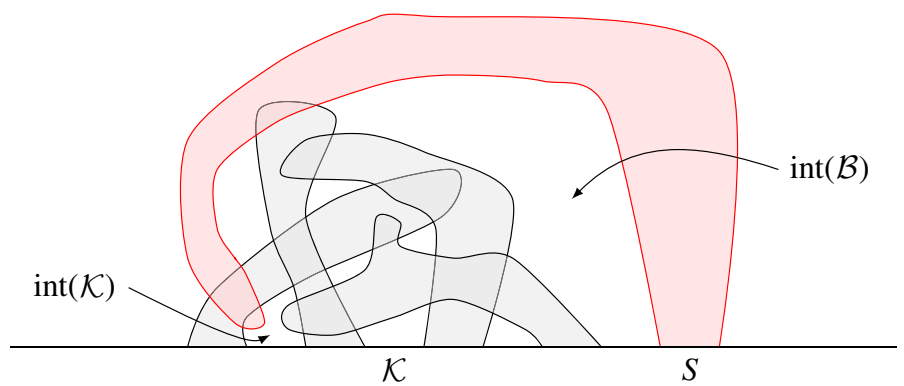

Fig. 3 A $k$-bracket $\mathcal{B}$ with $k$-clique $\mathcal{K}$ and support $S$

between the two $\prec$-least members of $\mathcal{K}$. A $k$-bracket in $\mathcal{F}$ is a subfamily of $\mathcal{F}$ consisting of a $k$-clique $\mathcal{K}$ and a set $S$ called the support such that $S \prec \mathcal{K}$ or $\mathcal{K} \prec S$ and $S \cap \operatorname{int}(\mathcal{K}) \neq \varnothing$. For such a $k$-bracket $\mathcal{B}$, we denote by $\operatorname{int}(\mathcal{B})$ the only arc-connected component of $(\mathbb{R} \times[0,+\infty)) \backslash \bigcup \mathcal{B}$ containing the part of the baseline between $S$ and $\mathcal{K}$ (see Fig. 3).

Lemma 6 Let $\mathcal{F}$ be a grounded family, $X, Y, Z \in \mathcal{F}$, and $X \cap Y \neq \emptyset$. Let $C_{1}$ and $C_{2}$ be any two distinct arc-connected components of $(\mathbb{R} \times[0,+\infty)) \backslash(X \cup Y)$. Let $z, z^{\prime} \in Z \cap C_{1}$. If every arc between $z$ and $z^{\prime}$ within $Z$ intersects $C_{2}$, then every such arc intersects both $X$ and $Y$.

Proof Suppose there is an arc $A \subset Z$ between $z$ and $z^{\prime}$ such that $A \cap X=\varnothing$ or $A \cap Y=\emptyset$. If $A \cap X=\emptyset$ and $A \cap Y=\emptyset$, then $A \subset C_{1}$, so $A \cap C_{2}=\emptyset$. Now, suppose $A \cap X=\emptyset$ and $A \cap Y \neq \emptyset$. Let $y$ and $y^{\prime}$ be respectively the first and last points of $A$ in $Y$. Since $Z \cap Y$ is arc-connected, there is an $\operatorname{arc} A^{\prime}$ in $Z$ that is simple with respect to $Y$ and goes along $A$ from $z$ to $y$, then to $y^{\prime}$ inside $Y$, and finally along $A$ to $z^{\prime}$. It follows that $A^{\prime} \subset C_{1} \cup Y$, so $A^{\prime} \cap C_{2}=\emptyset$. The case that $A \cap X \neq \emptyset$ and $A \cap Y=\varnothing$ is symmetric.

Corollary 7 Let $\mathcal{F}$ be a grounded family, $\mathcal{K}$ be a $k$-clique in $\mathcal{F}$, and $X \in \mathcal{F}$. If $x, y \in X \cap \operatorname{int}(\mathcal{K})$ (or $x, y \in X \cap \operatorname{ext}(\mathcal{K}))$ and every arc between $x$ and $y$ within $X$ intersects $\operatorname{ext}(\mathcal{K})(\operatorname{int}(\mathcal{K})$, respectively), then $X$ intersects every member of $\mathcal{K}$.

Proof Let $\mathcal{K}=\left\{K_{1}, \ldots, K_{k}\right\}$ and $K_{1} \prec \operatorname{int}(\mathcal{K}) \prec K_{2} \prec \cdots \prec K_{k}$. The statement follows directly from Lemma 6 and the fact that $\operatorname{int}(\mathcal{K})$ and $\operatorname{ext}(\mathcal{K})$ belong to distinct arc-connected components of $(\mathbb{R} \times[0,+\infty)) \backslash\left(K_{1} \cup K_{i}\right)$ for $2 \leqslant i \leqslant k$.

Corollary 8 Let $\mathcal{F}$ be a grounded family and $\mathcal{B}$ be a bracket in $\mathcal{F}$ with clique $\mathcal{K}$ and support $S$. Let $X \in \mathcal{F}$. If $X \cap \operatorname{int}(\mathcal{B}) \neq \emptyset$ and $X \cap \operatorname{ext}(\mathcal{B}) \neq \emptyset$, then $X$ intersects $S$ or every member of $\mathcal{K}$.

Proof Let $x \in X \cap \operatorname{int}(\mathcal{B})$ and $x^{\prime} \in X \cap \operatorname{ext}(\mathcal{B})$, and suppose $X \cap S=\emptyset$. By the Jordan curve theorem, every arc between $x$ and $x^{\prime}$ within $X$ must intersect $S \cup \operatorname{int}(\mathcal{K})$ and thus $\operatorname{int}(\mathcal{K})$. Since $x, x^{\prime} \in X \cap \operatorname{ext}(\mathcal{K})$, it follows from Corollary 7 that $X$ intersects every member of $\mathcal{K}$. 


\section{Proof of Proposition 3}

The proof goes by induction on $k$. Proposition 3 holds trivially for $k=1$ with $\xi_{1}=1$. Therefore, we assume that $k \geqslant 2$ and that the statement of the proposition holds for $k-1$. This context of the induction step is maintained throughout the entire remaining part of the paper. A typical application of the induction hypothesis looks as follows: if $\mathcal{F}$ is a grounded family with $\omega(\mathcal{F}) \leqslant k, \mathcal{G} \subset \mathcal{F}$, and there is $X \in \mathcal{F} \backslash \mathcal{G}$ intersecting all members of $\mathcal{G}$, then $\omega(\mathcal{G}) \leqslant k-1$ and thus $\chi(\mathcal{G}) \leqslant \xi_{k-1}$.

Define $\beta_{k}=8 k \xi_{k-1}^{2}, \delta_{k, k}=0, \delta_{k, j}=\beta_{k}+2 \delta_{k, j+1}+2 \xi_{k-1}\left(k \xi_{k-1}+k+2\right)+2$ for $k-1 \geqslant j \geqslant 0$, and finally $\xi_{k}=2^{k+2}\left(\delta_{k, 0}+2 \xi_{k-1}+1\right)$.

We say that a grounded set $X$ (a grounded family $\mathcal{X}$ ) is surrounded by a set $S$ if $X$ (every member of $\mathcal{X}$, respectively) is disjoint from $S \cup \operatorname{ext}(S)$. For a set $S$ and a grounded set $R$ such that base $(R)$ is surrounded by $S$, let cut $(R, S)$ denote the closure of the unique arc-connected component of $R \backslash S$ containing base $(R)$. For a set $S$ and a grounded family $\mathcal{R}$ of sets whose bases are surrounded by $S$, let $\operatorname{cut}(\mathcal{R}, S)=\{\operatorname{cut}(R, S): R \in \mathcal{R}\}$.

First, we present a technical lemma, which generalizes similar statements from [10] (Lemma 3.2) and [15] (Lemma 4.1), and which we will prove in Sect. 6. Loosely speaking, it says that one can color properly, with the number of colors bounded in terms of $k$, all the members of $\mathcal{F}$ surrounded by a set $S$ which intersect cut $(R, S)$ for any set $R \in \mathcal{F}$ intersecting $S$.

Lemma 9 Let $S$ be a compact set and $\mathcal{R} \cup \mathcal{D}$ be a grounded family with the following properties:

- the base of every member of $\mathcal{R}$ is surrounded by $S$,

- every member of $\mathcal{R}$ intersects $S$,

- $\mathcal{D}$ is surrounded by $S$,

- every member of $\mathcal{D}$ intersects $\bigcup \operatorname{cut}(\mathcal{R}, S)$,

- $\omega(\mathcal{R} \cup \mathcal{D}) \leqslant k$.

It follows that $\chi(\mathcal{D}) \leqslant \beta_{k}$.

Suppose for the sake of contradiction that there is a grounded family $\mathcal{F}$ with $\omega(\mathcal{F}) \leqslant k$ and $\chi(\mathcal{F})>\xi_{k}=2^{k+2}\left(\delta_{k, 0}+2 \xi_{k-1}+1\right)$. A repeated application of Lemma 5 yields a sequence of families $\mathcal{F}=\mathcal{F}_{k+1} \supset \mathcal{F}_{k} \supset \cdots \supset \mathcal{F}_{0}$ such that $\mathcal{F}_{i}$ is externally supported in $\mathcal{F}_{i+1}$ and $\chi\left(\mathcal{F}_{i}\right)>2^{i+1}\left(\delta_{k, 0}+2 \xi_{k-1}+1\right)$, for $0 \leqslant i \leqslant k$. The following claim is the core of the proof.

Claim 10 For $0 \leqslant j \leqslant k$, there are families $\mathcal{S}, \mathcal{G} \subset \mathcal{F}_{j}$ and sets $S_{1}, \ldots, S_{j} \in \mathcal{S}$ with the following properties:

(i) $\mathcal{G}$ is surrounded by $\cup \mathcal{S}$,

(ii) $\chi(\mathcal{G})>\delta_{k, j}$,

(iii) the sets $S_{1}, \ldots, S_{j}$ pairwise intersect,

(iv) every member of $\mathcal{F}$ intersecting $\operatorname{ext}(\mathcal{S})$ and some member of $\mathcal{G}$ also intersects each of $S_{1}, \ldots, S_{j}$. 
Proof The proof goes by induction on $j$. First, let $j=0$. Apply Lemma 4 to find $\mathcal{H} \subset \mathcal{F}_{0}$ such that $\chi(\mathcal{H})>1$ and for any intersecting $H_{1}, H_{2} \in \mathcal{H}$ we have $\chi\left(\mathcal{F}_{0}\left(H_{1}, H_{2}\right)\right)>\delta_{k, 0}+2 \xi_{k-1}$. Since $\chi(\mathcal{H})>1$, such two intersecting $H_{1}, H_{2} \in \mathcal{H}$ exist. Let $\mathcal{S}=\left\{H_{1}, H_{2}\right\}$ and $\mathcal{G}$ be the family of those members of $\mathcal{F}_{0}\left(H_{1}, H_{2}\right)$ that are disjoint from $H_{1} \cup H_{2}$. It is clear that (i) holds. Since the members of $\mathcal{F}_{0}\left(H_{1}, H_{2}\right)$ intersecting $H_{1} \cup H_{2}$ have chromatic number at most $2 \xi_{k-1}$, we have $\chi(\mathcal{G})>\delta_{k, 0}$, so (ii) holds. The conditions (iii) and (iv) are satisfied vacuously.

Now, assume that $j \geqslant 1$ and the claim holds for $j-1$, that is, there are families $\mathcal{S}^{\prime}, \mathcal{G}^{\prime} \subset \mathcal{F}_{j-1}$ and sets $S_{1}, \ldots, S_{j-1} \in \mathcal{S}^{\prime}$ satisfying (i)-(iv). Let

$$
\begin{aligned}
& \mathcal{R}=\left\{R \in \mathcal{F}: \text { base }(R) \text { is surrounded by } \bigcup \mathcal{S}^{\prime} \text { and } R \cap \bigcup \mathcal{S}^{\prime} \neq \emptyset\right\}, \\
& \mathcal{D}=\left\{D \in \mathcal{G}^{\prime}: D \cap \bigcup \operatorname{cut}\left(\mathcal{R}, \bigcup \mathcal{S}^{\prime}\right) \neq \emptyset\right\} .
\end{aligned}
$$

It follows from Lemma 9 that $\chi(\mathcal{D}) \leqslant \beta_{k}$ and thus $\chi\left(\mathcal{G}^{\prime} \backslash \mathcal{D}\right)>2 \delta_{k, j}+2 \xi_{k-1}\left(k \xi_{k-1}+\right.$ $k+2)+2$. Since the chromatic number of a graph is the maximum chromatic number of its connected component, there is $\mathcal{G}^{\prime \prime} \subset \mathcal{G}^{\prime} \backslash \mathcal{D}$ such that the intersection graph of $\mathcal{G}^{\prime \prime}$ is connected and $\chi\left(\mathcal{G}^{\prime \prime}\right)>2 \delta_{k, j}+2 \xi_{k-1}\left(k \xi_{k-1}+k+2\right)+2$. Partition $\mathcal{G}^{\prime \prime}$ into three subfamilies $\mathcal{X}, \mathcal{Y}, \mathcal{Z}$ so that $\mathcal{X} \prec \mathcal{Y} \prec \mathcal{Z}$ and $\chi(\mathcal{X})=\chi(\mathcal{Z})=\delta_{k, j}+(k+1) \xi_{k-1}+1$. It follows that $\chi(\mathcal{Y})>2 \xi_{k-1}\left(k \xi_{k-1}+1\right)$. Apply Lemma 4 to find $\mathcal{H} \subset \mathcal{Y}$ such that $\chi(\mathcal{H})>\xi_{k-1}$ and for any intersecting $H_{1}, H_{2} \in \mathcal{H}$ we have $\chi\left(\mathcal{Y}\left(H_{1}, H_{2}\right)\right)>k \xi_{k-1}$. Since $\chi(\mathcal{H})>\xi_{k-1}$, there is a $k$-clique $\mathcal{K} \subset \mathcal{H}$. The members of $\mathcal{Y}$ intersecting $\bigcup \mathcal{K}$ have chromatic number at most $k \xi_{k-1}$, so there is $P \in \mathcal{Y}$ that is contained in $\operatorname{int}(\mathcal{K})$. Since $\mathcal{F}_{j-1}$ is externally supported in $\mathcal{F}_{j}$, there is $S_{j} \in \mathcal{F}_{j}$ such that $S_{j} \cap P \neq \varnothing$ and $S_{j} \cap \operatorname{ext}\left(\mathcal{S}^{\prime}\right) \supset S_{j} \cap \operatorname{ext}\left(\mathcal{F}_{j-1}\right) \neq \emptyset$. Therefore, since $\mathcal{S}^{\prime}$ and $\mathcal{G}^{\prime}$ satisfy (iv), $S_{j}$ intersects each of $S_{1}, \ldots, S_{j-1}$ and thus (iii) holds for $S_{1}, \ldots, S_{j}$.

We show that $S_{j} \prec \mathcal{G}^{\prime \prime}$ or $\mathcal{G}^{\prime \prime} \prec S_{j}$. Suppose that neither of these holds. It follows that base $\left(S_{j}\right)$ is surrounded by $\bigcup \mathcal{S}^{\prime}$, which yields $S_{j} \in \mathcal{R}$. Moreover, base $\left(S_{j}\right)$ is surrounded by $\cup \mathcal{G}^{\prime \prime}$, as the intersection graph of $\mathcal{G}^{\prime \prime}$ is connected. Therefore, we have $\operatorname{cut}\left(S_{j}, \bigcup \mathcal{S}^{\prime}\right) \cap \bigcup \mathcal{G}^{\prime \prime} \neq \emptyset$, so there is $X \in \mathcal{G}^{\prime \prime}$ such that $X \cap \operatorname{cut}\left(S_{j}, \bigcup \mathcal{S}^{\prime}\right) \neq \emptyset$. This means that $X \in \mathcal{D}$, which contradicts the definition of $\mathcal{G}^{\prime \prime}$.

Now, we have $S_{j} \cap \operatorname{int}(\mathcal{K}) \supset S_{j} \cap P \neq \varnothing$ and $S_{j} \prec \mathcal{K}$ or $\mathcal{K} \prec S_{j}$, so the $k$-clique $\mathcal{K}$ and the support $S_{j}$ form a $k$-bracket. Let $\mathcal{S}=\mathcal{S}^{\prime} \cup \mathcal{K} \cup\left\{S_{j}\right\}$. If $S_{j} \prec \mathcal{G}^{\prime \prime}$, then $S_{j} \prec \mathcal{X} \prec \mathcal{K}$. In this case, let $\mathcal{G}$ be the family of those members of $\mathcal{X}$ that are disjoint from $\bigcup \mathcal{K} \cup S_{j}$. It is clear that (i) holds. Since $\chi(\mathcal{X})>\delta_{k, j}+(k+1) \xi_{k-1}$ and the members of $\mathcal{X}$ intersecting $\cup \mathcal{K} \cup S_{j}$ have chromatic number at most $(k+1) \xi_{k-1}$, we have $\chi(\mathcal{G})>\delta_{k, j}$, so (ii) holds. Since $\operatorname{ext}(\mathcal{S}) \subset \operatorname{ext}\left(\mathcal{K} \cup\left\{S_{j}\right\}\right)$, it follows from Corollary 8 that every member of $\mathcal{F}$ intersecting $\operatorname{ext}(\mathcal{S})$ and some member of $\mathcal{G}$ intersects $S_{j}$. Hence (iv) holds. If $\mathcal{G}^{\prime \prime} \prec S_{j}$, then let $\mathcal{G}$ be the family of those members of $\mathcal{Z}$ that are disjoint from $\bigcup \mathcal{K} \cup S_{j}$. An analogous argument shows that (i), (ii), and (iv) are satisfied.

Let $\mathcal{S}, \mathcal{G}$, and $S_{1}, \ldots, S_{k}$ be as guaranteed by Claim 10 for $j=k$. By (ii), we have $\chi(\mathcal{G})>0$, so there is $P \in \mathcal{G}$. Since $\mathcal{F}_{k}$ is externally supported in $\mathcal{F}$, there is $S_{k+1} \in \mathcal{F}$ such that $S_{k+1} \cap P \neq \varnothing$ and $S_{k+1} \cap \operatorname{ext}(\mathcal{S}) \neq \varnothing$. By (iii) and (iv), we conclude that $S_{1}, \ldots, S_{k+1}$ pairwise intersect. This contradicts the assumption that $\omega(\mathcal{F}) \leqslant k$, thus completing the proof of Proposition 3. 


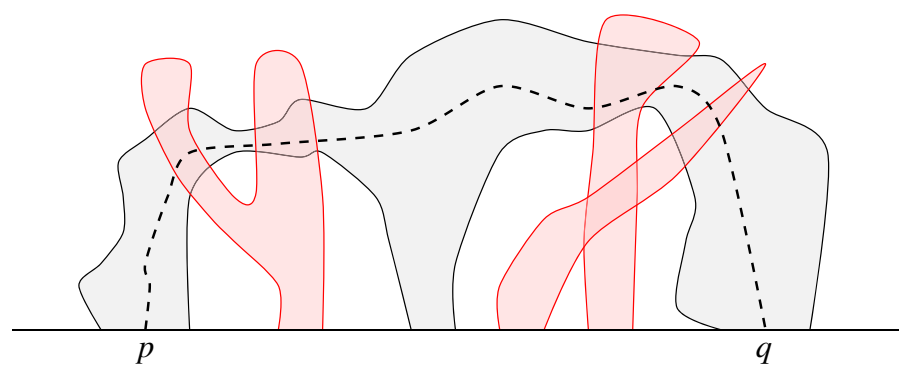

Fig. 4 The setting of the proof of Lemma 9: the set $S$ with a dashed $\operatorname{arc} S^{\prime}$ and three sets from $\mathcal{R}$

\section{Proof of Lemma 9}

The proof of Lemma 9 goes along similar lines to the proof of Lemma 4.1 in [15]. Since $\mathcal{D}$ is surrounded by $S$, there is an $\operatorname{arc} S^{\prime} \subset S$ such that $\mathcal{D}$ is surrounded by $S^{\prime}$. We can assume without loss of generality that base $\left(S^{\prime}\right)=\{p, q\}$ for some points $p$ and $q$ on the baseline such that $p \prec \mathcal{D} \prec q$. For every $R \in \mathcal{R}$ we have $\operatorname{cut}(R, S) \subset \operatorname{cut}\left(R, S^{\prime}\right)$. Hence every member of $\mathcal{D}$ intersects $\bigcup \operatorname{cut}\left(R, S^{\prime}\right)$ (see Fig. 4).

Claim $11 \chi\left(\operatorname{cut}\left(\mathcal{R}, S^{\prime}\right)\right) \leqslant k$.

Proof Consider a relation $<$ on $\operatorname{cut}\left(\mathcal{R}, S^{\prime}\right)$ defined as follows: $R_{1}^{\prime}<R_{2}^{\prime}$ if and only if $R_{1}^{\prime} \prec R_{2}^{\prime}$ and $R_{1}^{\prime} \cap R_{2}^{\prime}=\emptyset$. It is clear that $<$ is irreflexive and antisymmetric. It is also transitive, which follows from the fact that if $R_{1}^{\prime}, R_{2}^{\prime}, R_{3}^{\prime} \in \mathcal{R}, R_{1}^{\prime} \prec R_{2}^{\prime} \prec R_{3}^{\prime}$, and $R_{1}^{\prime} \cap R_{3}^{\prime} \neq \emptyset$, then $R_{2}^{\prime} \cap\left(R_{1}^{\prime} \cup R_{3}^{\prime}\right) \neq \emptyset$. Therefore, $<$ is a strict partial order. The intersection graph of $\operatorname{cut}\left(\mathcal{R}, S^{\prime}\right)$ is the incomparability graph of $<$, so it is perfect, which implies $\chi\left(\operatorname{cut}\left(\mathcal{R}, S^{\prime}\right)\right)=\omega\left(\operatorname{cut}\left(\mathcal{R}, S^{\prime}\right)\right) \leqslant k$.

By Claim 11, there is a coloring $\phi$ of $\mathcal{R}$ with $k$ colors such that for any $R_{1}, R_{2} \in \mathcal{R}$ with $\phi\left(R_{1}\right)=\phi\left(R_{2}\right)$, we have $\operatorname{cut}\left(R_{1}, S^{\prime}\right) \cap \operatorname{cut}\left(R_{2}, S^{\prime}\right)=\emptyset$. For a color $c$, let $\mathcal{R}^{c}=\{R \in \mathcal{R}: \phi(R)=c\}$ and $\mathcal{D}^{c}=\left\{D \in \mathcal{D}: D \cap \bigcup \operatorname{cut}\left(\mathcal{R}^{c}, S^{\prime}\right) \neq \emptyset\right\}$. We are going to show that $\chi\left(\mathcal{D}^{c}\right) \leqslant 8 \xi_{k-1}^{2}$. Once this is obtained, we will have $\chi(\mathcal{D}) \leqslant$ $\sum_{c} \chi\left(\mathcal{D}^{c}\right) \leqslant 8 k \xi_{k-1}^{2}=\beta_{k}$.

Since the sets cut $\left(R, S^{\prime}\right)$ for $R \in \mathcal{R}^{c}$ are pairwise disjoint, the curve $S^{\prime}$ and the families $\operatorname{cut}\left(\mathcal{R}^{c}, S^{\prime}\right)$ and $\mathcal{D}^{c}$ satisfy the assumptions of Lemma 9. To simplify the notation, we assume for the remainder of the proof that $S=S^{\prime}, R=\operatorname{cut}\left(R, S^{\prime}\right)$ for every $R \in \mathcal{R}^{c}, \mathcal{R}=\operatorname{cut}\left(\mathcal{R}^{c}, S^{\prime}\right)$, and $\mathcal{D}=\mathcal{D}^{c}$. By Jordan-Schönflies theorem, the segment $p q$ and the arc $S$ form a Jordan curve which is the boundary of a set $J$ homeomorphic to a closed disc. In this new setting, $S$ is an arc and $\mathcal{R} \cup \mathcal{D}$ is a grounded family with the following properties:

- the base of every member of $\mathcal{R}$ is surrounded by $S$,

- every member of $\mathcal{R}$ is contained in $J$ and intersects $S$,

- the members of $\mathcal{R}$ are pairwise disjoint,

- $\mathcal{D}$ is surrounded by $S$,

- every member of $\mathcal{D}$ intersects $\bigcup \mathcal{R}$,

- $\omega(\mathcal{R} \cup \mathcal{D}) \leqslant k$. 
We enumerate the members of $\mathcal{R}$ as $R_{1}, \ldots, R_{m}$ in the $\prec$-order, that is, so that $R_{1} \prec$ $\cdots \prec R_{m}$. We are going to show that $\chi(\mathcal{D}) \leqslant 8 \xi_{k-1}^{2}$.

Claim 12 For $1 \leqslant i<j \leqslant m$, any arc in $J$ between $R_{i}$ and $R_{j}$ intersects all $R_{i+1}, \ldots, R_{j-1}$.

Proof Let $A$ be an arc in $J$ between points $x_{i} \in R_{i}$ and $x_{j} \in R_{j}$. For any $R \in$ $\left\{R_{i+1}, \ldots, R_{j-1}\right\}$, base $(R)$ is surrounded by $R_{i} \cup A \cup R_{j}$ and we have $R \cap\left(R_{i} \cup A \cup\right.$ $\left.R_{j}\right) \neq \emptyset$, as $R \cap S \neq \emptyset$. Since $R$ is disjoint from $R_{i}$ and $R_{j}$, we have $R \cap A \neq \emptyset$.

A point $x \in J$ is a neighbor of $R_{i}$ if there is an arc in $J$ between $x$ and $R_{i}$ disjoint from all $R_{1}, \ldots, R_{m}$ except $R_{i}$. It follows from Claim 12 that each point in $J$ is a neighbor of at most two consecutive sets of $R_{1}, \ldots, R_{m}$. For $1 \leqslant i<m$, let $I_{i}$ denote the set of points in $J$ that are neighbors of $R_{i}$ and $R_{i+1}$.

Claim 13 Any arc-connected subset of $J$ intersects an interval of sets in the sequence $R_{1}, I_{1}, R_{2}, \ldots, I_{m-1}, R_{m}$.

Proof Let $X$ be an arc-connected subset of $J$. First, we show that if $X$ intersects $R_{i}$ and $R_{i+1}$, then it also intersects $I_{i}$. This is guaranteed by the compactness of $R_{i}$ and $R_{i+1}$. Indeed, take a $\subset$-minimal arc in $J$ between $R_{i}$ and $R_{i+1}$. By Claim 12, the interior of this arc is disjoint from all $R_{1}, \ldots, R_{m}$ and therefore must lie in $I_{i}$.

Now, let $i$ be the least index such that $X \cap\left(R_{i} \cup I_{i}\right) \neq \varnothing$ and $j$ be the greatest index such that $X \cap\left(I_{j-1} \cup R_{j}\right) \neq \emptyset$. Let $A$ be an arc in $X$ between points $x_{i} \in X \cap\left(R_{i} \cup I_{i}\right)$ and $x_{j} \in X \cap\left(I_{j-1} \cup R_{j}\right)$. Since $x_{i}$ is a neighbor of $R_{i}$, there is an $\operatorname{arc} A_{i} \subset J$ between $x_{i}$ and $R_{i}$ disjoint from all $R_{1}, \ldots, R_{m}$ except $R_{i}$. Similarly, since $x_{j}$ is a neighbor of $R_{j}$, there is an $\operatorname{arc} A_{j} \subset J$ between $x_{j}$ and $R_{j}$ disjoint from all $R_{1}, \ldots, R_{m}$ except $R_{j}$. There is an $\operatorname{arc} \bar{A} \subset A_{i} \cup A \cup A_{j}$ between $R_{i}$ and $R_{j}$. By Claim $12, \bar{A}$ intersects all $R_{i+1}, \ldots, R_{j-1}$. But $R_{i+1}, \ldots, R_{j-1}$ are disjoint from $A_{i}$ and $A_{j}$, hence they intersect $A$.

For convenience, define $I_{0}=I_{m}=\emptyset$. For $D \in \mathcal{D}$, define leftclip $(D)=D \backslash I_{i}$ and $\operatorname{rightclip}(D)=D \backslash I_{j}$, where $i$ and $j$ are chosen so that $R_{i+1}$ is the first and $R_{j}$ is the last member of $\mathcal{R}$ intersecting $D$ (see Fig. 5). This definition extends to families $\mathcal{M} \subset \mathcal{D}$ : $\operatorname{leftclip}(\mathcal{M})=\{\operatorname{leftclip}(M): M \in \mathcal{M}\}$ and $\operatorname{rightclip}(\mathcal{M})=\{\operatorname{rightclip}(M): M \in \mathcal{M}\}$.

Claim 14 leftclip $(\mathcal{D})$ and $\operatorname{rightclip}(\mathcal{D})$ are simple families of compact arc-connected sets.

Proof We present the proof only for $\operatorname{leftclip}(\mathcal{D})$, as for $\operatorname{rightclip}(\mathcal{D})$ it is analogous. The sets $I_{1}, \ldots, I_{m-1}$ are open in $J$, as they are arc-connected components of the set $J \backslash \bigcup \mathcal{R}$, which is open in $J$. Each member of leftclip $(\mathcal{D})$ is a difference of a compact set in $\mathcal{D}$ and one of $I_{1}, \ldots, I_{m-1}$ and thus is compact as well.

To prove that leftclip $(\mathcal{D})$ is simple and consists of arc-connected sets, we need to show that $\bigcap \operatorname{leftclip}(\mathcal{M})$ is arc-connected for any $\mathcal{M} \subset \mathcal{D}$. Let $x, y \in$ $\bigcap$ leftclip $(\mathcal{M}) \subset \bigcap \mathcal{M}$. Since $\bigcap \mathcal{M}$ is arc-connected, Lemma 2 provides us with an arc $A \subset \bigcap \mathcal{M}$ between $x$ and $y$ that is simple with respect to $\mathcal{R}$. It suffices to show $A \subset \operatorname{leftclip}(M)$ for each $M \in \mathcal{M}$. To this end, fix $M \in \mathcal{M}$ 


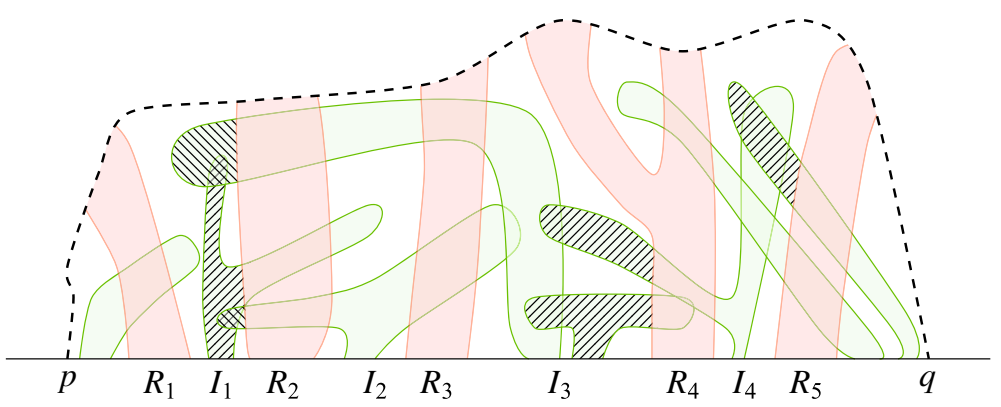

Fig. 5 A dashed arc $S$, sets from $\mathcal{R}$ spanned between the baseline and $S$, and sets from $\mathcal{D}$ with $D \backslash$ leftclip $(D)$ marked for each $D \in \mathcal{D}$

and let $R_{i}$ be the $\prec$-least member of $\mathcal{R}$ intersecting $M$. Suppose there is a point $z \in A \cap(M \backslash \operatorname{leftclip}(M)) \subset I_{i-1}$. Since $x, y \in \operatorname{leftclip}(M) \subset \bigcup_{j=i}^{m}\left(R_{j} \cup I_{j}\right)$, it follows from Claim 13 that the parts of $A$ from $x$ to $z$ and from $z$ to $y$ intersect $R_{i}$. This and $z \notin R_{i}$ contradict the simplicity of $A$ with respect to $R_{i}$.

Claim 15 leftclip $(\mathcal{D})$ and $\operatorname{rightclip}(\mathcal{D})$ have clique number at most $k-1$.

Proof Again, we present the proof only for leftclip $(\mathcal{D})$. Let $\mathcal{K}$ be a clique in leftclip $(\mathcal{D})$. By Claim 14, each member of $\mathcal{K}$ is arc-connected. Therefore, by Claim 13, each member of $\mathcal{K}$ intersects an interval of sets in the sequence $R_{1}, I_{1}, \ldots, R_{m-1}, I_{m-1}, R_{m}$, the first set in this interval being of the form $R_{j}$. Since the members of $\mathcal{K}$ pairwise intersect, these intervals also pairwise intersect, which implies that they all contain a common $R_{j}$. Thus $\mathcal{K} \cup\left\{R_{j}\right\}$ is a clique, and $\omega(\mathcal{R} \cup \mathcal{D}) \leqslant k$ yields $|\mathcal{K}| \leqslant k-1$.

Recall that we need to show $\chi(\mathcal{D}) \leqslant 8 \xi_{k-1}^{2}$. Define

- $\mathcal{D}^{L}=\{X \in \mathcal{D}:$ base $(\operatorname{leftclip}(X)) \neq \emptyset\}$,

- $\mathcal{D}^{R}=\{X \in \mathcal{D}$ : base $(\operatorname{rightclip}(X)) \neq \emptyset\}$.

Since each member of $\mathcal{D}$ intersects at least one of $R_{1}, \ldots, R_{m}$, we have $\mathcal{D}^{L} \cup \mathcal{D}^{R}=\mathcal{D}$. Therefore, it is enough to show that $\chi\left(\mathcal{D}^{L}\right) \leqslant 4 \xi_{k-1}^{2}$ and $\chi\left(\mathcal{D}^{R}\right) \leqslant 4 \xi_{k-1}^{2}$. We only present the proof of $\chi\left(\mathcal{D}^{R}\right) \leqslant 4 \xi_{k-1}^{2}$, as the proof of the other inequality is analogous.

By Claims 14 and $15, \operatorname{rightclip}\left(\mathcal{D}^{R}\right)$ is a grounded family with clique number at most $k-1$. This and the induction hypothesis yield $\chi\left(\operatorname{rightclip}\left(\mathcal{D}^{R}\right)\right) \leqslant \xi_{k-1}$. We fix a coloring $\phi^{R}$ of $\mathcal{D}^{R}$ with $\xi_{k-1}$ colors so that $\phi^{R}(X) \neq \phi^{R}(Y)$ for any $X, Y \in \mathcal{D}^{R}$ with $\operatorname{rightclip}(X) \cap \operatorname{rightclip}(Y) \neq \emptyset$. Let $\mathcal{M} \subset \mathcal{D}^{R}$ be a family of sets having the same color in $\phi^{R}$. In particular, we have $\operatorname{rightclip}(X) \cap \operatorname{rightclip}(Y)=\emptyset$ for any $X, Y \in \mathcal{M}$. It remains to prove that $\chi(\mathcal{M}) \leqslant 4 \xi_{k-1}$.

We show how to construct a coloring $\phi^{L}$ of $\mathcal{M}$ with $\xi_{k-1}$ colors such that $\operatorname{leftclip}(X) \cap \operatorname{leftclip}(Y)=\emptyset$ for any $X, Y \in \mathcal{M}$ with $\phi^{L}(X)=\phi^{L}(Y)$. We exploit the fact that members of $\mathcal{M}$ have pairwise disjoint intersections with each $R_{i}$ to simplify the topology of $\mathcal{M}$ and $R_{1}, \ldots, R_{m}$. Recall that $S$ is an arc with $\operatorname{base}(S)=\{p, q\}$. For $1 \leqslant i \leqslant m$, by Lemma 2 , there is an arc $Q_{i} \subset R_{i}$ between base $\left(R_{i}\right)$ and $R_{i} \cap S$ that is simple with respect to $\mathcal{M}$. We assume without loss of generality that $\operatorname{base}\left(Q_{i}\right)=\left\{u_{i}\right\}$ and $Q_{i} \cap S=\left\{v_{i}\right\}$. The points $v_{1}, \ldots, v_{m}$ occur in this order on 
$S$ as moving from $p$ to $q$. Moreover, the $\operatorname{arcs} Q_{1}, \ldots, Q_{m}$ partition $J$ into $m+1$ sets $J_{0}, \ldots, J_{m}$, each homeomorphic to a closed disc, so that $J_{i-1} \cap J_{i}=Q_{i}$ for $1 \leqslant i \leqslant m$. It is clear that each arc-connected subset of $J$ intersects an interval of sets in the sequence $J_{0}, Q_{1}, J_{1}, \ldots, Q_{m}, J_{m}$. Since $Q_{1}, \ldots, Q_{m}$ are simple with respect to $\mathcal{M}$, so are $J_{0}, \ldots, J_{m}$.

Since the sets $J_{0}, \ldots, J_{m}$ are homeomorphic to a closed disc and so are rectangles with bottom sides base $\left(J_{0}\right), \ldots$, base $\left(J_{m}\right)$, there are homeomorphisms $\mu_{0}, \ldots, \mu_{m}$ such that

- $\mu_{i}$ is constant on base $\left(J_{i}\right)$ and maps $J_{i}$ onto a rectangle with bottom side base $\left(J_{i}\right)$ for $0 \leqslant i \leqslant m$,

- $\mu_{i-1}$ and $\mu_{i}$ agree on $Q_{i}$ for $1 \leqslant i \leqslant m$.

Thus $\mu_{0} \cup \cdots \cup \mu_{m}$ is a homeomorphism between $J$ and a rectangle with bottom side base $(J)$, and it extends to a homeomorphism $\mu$ of $\mathbb{R} \times[0,+\infty)$ whose restriction to each $J_{i}$ is $\mu_{i}$. Let $\tau_{0}, \ldots, \tau_{m}$ be horizontal translations such that $\tau_{0}(u) \prec \cdots \prec$ $\tau_{m}(u)$ for a point $u$ on the baseline. Let $x_{1}, \ldots, x_{m}$ be the $x$-coordinates of the points $u_{1}, \ldots, u_{m}$, respectively, so that $u_{i}=\left(x_{i}, 0\right)$ for $1 \leqslant i \leqslant m$. Define

- $\hat{J}_{0}=\mu^{-1}\left(\left(-\infty, x_{1}\right] \times[0,+\infty)\right)$,

- $\hat{J}_{i}=\mu^{-1}\left(\left[x_{i}, x_{i+1}\right] \times[0,+\infty)\right)$ for $1 \leqslant i<m$,

- $\hat{J}_{m}=\mu^{-1}\left(\left[x_{m},+\infty\right) \times[0,+\infty)\right)$,

- $\hat{Q}_{i}=\mu^{-1}\left(\left\{x_{i}\right\} \times[0,+\infty)\right)$ for $1 \leqslant i \leqslant m$,

- $\sigma_{i}=\tau_{i} \circ \mu$ for $0 \leqslant i \leqslant m$.

Note that $J_{i} \subset \hat{J}_{i}$ and $Q_{i} \subset \hat{Q}_{i}$. For a set $X$, define

$$
\begin{array}{r}
X^{\star}=\sigma_{0}\left(X \cap \hat{J}_{0}\right) \cup\left[\sigma_{0}\left(X \cap \hat{Q}_{1}\right), \sigma_{1}\left(X \cap \hat{Q}_{1}\right)\right] \cup \sigma_{1}\left(X \cap \hat{J}_{1}\right) \cup \cdots \\
\cup\left[\sigma_{m-1}\left(X \cap \hat{Q}_{m}\right), \sigma_{m}\left(X \cap \hat{Q}_{m}\right)\right] \cup \sigma_{m}\left(X \cap \hat{J}_{m}\right)
\end{array}
$$

where $[Y, Z]$ denotes the rectangle with left side $Y$ and right side $Z$. It is clear that the map $X \mapsto X^{\star}$ preserves compactness and arc-connectedness and is compatible with unions and intersections. In particular, $\mathcal{M}^{\star}=\left\{X^{\star}: X \in \mathcal{M}\right\}$ is a grounded family with intersection graph isomorphic to that of $\mathcal{M}$ (see Fig. 6).

In the remainder of the proof, we will deal with $\mathcal{M}^{\star}$ and $R_{1}^{\star}, \ldots, R_{m}^{\star}$, but for simplicity we relabel them to $\mathcal{M}$ and $R_{1}, \ldots, R_{m}$, respectively. We also relabel $I_{0}^{\star}, \ldots, I_{m}^{\star}$ to $I_{0}, \ldots, I_{m}, Q_{1}^{\star}, \ldots, Q_{m}^{\star}$ to $Q_{1}, \ldots, Q_{m}, S^{\star}$ to $S, J^{\star}$ to $J$, and $\sigma_{0}\left(J_{0}\right), \ldots, \sigma_{m}\left(J_{m}\right)$ to $J_{0}, \ldots, J_{m}$. The following properties clearly follow:

- $J_{0}, \ldots, J_{m}$ are pairwise disjoint rectangles,

- $Q_{i}$ is a rectangle whose left side is the right side of $J_{i-1}$ and whose right side is the left side of $J_{i}$, for $1 \leqslant i \leqslant m$,

- every arc-connected subset of $J$ intersects an interval of sets in the sequence $J_{0}, Q_{1}, J_{1}, \ldots, Q_{m}, J_{m}$,

- $Q_{i} \subset R_{i}$ for $1 \leqslant i \leqslant m$,

- the intersection of any member of $\mathcal{M}$ with any $J_{i}$ is arc-connected,

- the intersection of any member of $\mathcal{M}$ with any $Q_{i}$ is a rectangle or horizontal segment spanning the entire width of $Q_{i}$. 

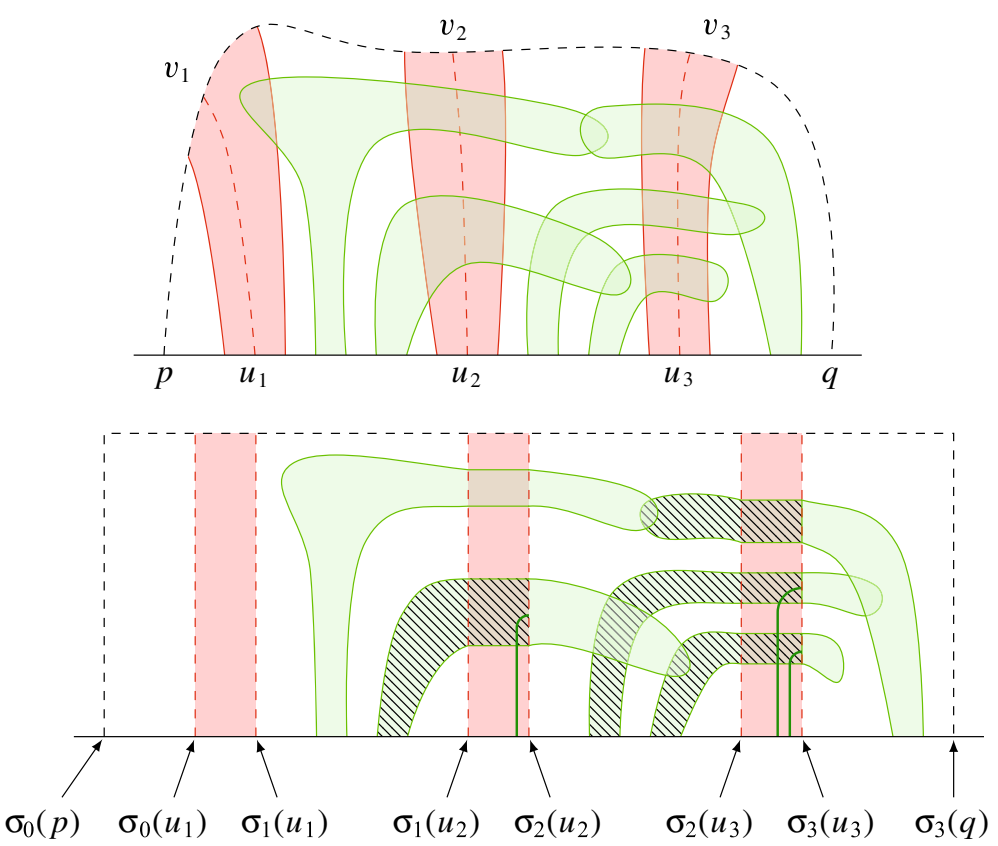

Fig. 6 The transformation $X \mapsto X^{\star}$; top: a family $\mathcal{M}$ before transformation; bottom: the families $\mathcal{M}^{\star}$ (including the marked regions) and $\mathcal{M}^{+}$(excluding the marked regions), and connections of the sets in $\mathcal{M}^{\star}$ to the baseline

Claim 16 There is a grounded family $\mathcal{M}^{\prime}$ with intersection graph isomorphic to that of $\operatorname{leftclip}(\mathcal{M})$.

Proof Let $\mathcal{M}_{i}=\left\{X \in \mathcal{M}: X \cap R_{i} \neq \emptyset\right\}$ for $1 \leqslant i \leqslant m$. It follows that leftclip $(X)=$ $X \backslash I_{i}$ for every $X \in \mathcal{M}_{i+1} \backslash \mathcal{M}_{i}$ and $1 \leqslant i<m$. We can assume without loss of generality that each member of $\mathcal{M}_{i+1} \backslash \mathcal{M}_{i}$ intersects $Q_{i+1}$ for $1 \leqslant i<m$, as those that do not are isolated vertices in the intersection graph of $\operatorname{leftclip}(\mathcal{M})$ and thus do not influence the existence of $\mathcal{M}^{\prime}$.

For $X \in \mathcal{M}_{i+1} \backslash \mathcal{M}_{i}$ and $1 \leqslant i<m$, let $X^{+}$denote the part of $X$ to the right of $Q_{i+1}$ including the right side of $Q_{i+1}$, that is, $X^{+}=X \cap\left(J_{i+1} \cup Q_{i+2} \cup J_{i+2} \cup \cdots \cup Q_{m} \cup J_{m}\right)$. It follows that $X^{+} \subset$ leftclip $(X) \subset X^{+} \cup R_{i+1}$ and $X^{+}$is compact and arc-connected. For $X \in \mathcal{M}_{1}$, let $X^{+}=X=\operatorname{leftclip}(X)$. Let $\mathcal{M}^{+}=\left\{X^{+}: X \in \mathcal{M}\right\}$. Since the intersections of the members of $\operatorname{leftclip}(\mathcal{M})$ with each $R_{i+1}$ are pairwise disjoint, the intersection graphs of $\mathcal{M}^{+}$and $\operatorname{leftclip}(\mathcal{M})$ are isomorphic. We show how to extend the sets $X^{+}$for which base $\left(X^{+}\right)=\operatorname{base}(\operatorname{leftclip}(X))=\emptyset$ to connect them to the baseline without creating any new intersections (see Fig. 6).

Let $1 \leqslant i<m, X \in \mathcal{M}_{i+1} \backslash \mathcal{M}_{i}$, and base $\left(X^{+}\right)=\emptyset$. Thus base $(X) \subset \operatorname{base}\left(J_{i}\right)$. For every $Y \in \mathcal{M}_{i}$, it is an immediate consequnce of the Jordan curve theorem and arc-connectedness of $X \cap J_{i}$ and $\left(Y \cup R_{i}\right) \cap J_{i}$ that $Y \cap Q_{i+1}$ is empty or lies above $X \cap Q_{i+1}$. Therefore, we can connect $X^{+}$to base $\left(Q_{i+1}\right)$ by an arc inside $Q_{i+1}$ that is disjoint from any other $Y^{+} \in \mathcal{M}^{+}$. Moreover, all these arcs for $X \in \mathcal{M}_{i+1} \backslash \mathcal{M}_{i}$ with base $\left(X^{+}\right)=\emptyset$ can be drawn so that they are pairwise disjoint. Doing so for all 
$i$ with $1 \leqslant i<m$, we transform $\mathcal{M}^{+}$into a grounded family $\mathcal{M}^{\prime}$ with intersection graph isomorphic to the intersection graph of $\mathcal{M}^{+}$and hence of leftclip $(\mathcal{M})$.

Claim 16 allows us to use the induction hypothesis on $\operatorname{leftclip}(\mathcal{M})$ to obtain a coloring $\phi^{L}$ of $\mathcal{M}$ with $\xi_{k-1}$ colors such that leftclip $(X) \cap \operatorname{leftclip}(Y)=\emptyset$ for any $X, Y \in \mathcal{M}$ with $\phi^{L}(X)=\phi^{L}(Y)$. Let $\mathcal{N} \subset \mathcal{M}$ be a family of sets having the same color in $\phi^{L}$. In particular, we have $\operatorname{leftclip}(X) \cap \operatorname{leftclip}(Y)=\varnothing$ and $\operatorname{rightclip}(X) \cap$ $\operatorname{rightclip}(Y)=\varnothing$ for any $X, Y \in \mathcal{N}$. The following claim completes the proof of Lemma 9. The planarity argument used in the proof applies the ideas of McGuinness [10].

Claim $17 \chi(\mathcal{N}) \leqslant 4$.

Proof Define

- $\mathcal{N}^{L}=\{X \in \mathcal{N}: X \backslash \operatorname{leftclip}(X) \neq \emptyset\}$,

- $\mathcal{N}^{R}=\{X \in \mathcal{N}: X \backslash \operatorname{rightclip}(X) \neq \emptyset\}$,

- $\mathcal{N}_{i}^{L}=\left\{X \in \mathcal{N}^{L}: X \backslash \operatorname{leftclip}(X) \subset J_{i}\right\}$ for $1 \leqslant i<m$,

- $\mathcal{N}_{i}^{R}=\left\{X \in \mathcal{N}^{R}: X \backslash \operatorname{rightclip}(X) \subset J_{i}\right\}$ for $1 \leqslant i<m$,

- $\mathcal{N}_{i}=\mathcal{N}_{i}^{L} \cup \mathcal{N}_{i}^{R}$,

- $C_{X}^{L}$ to be the arc-connected component of $\left(\bigcup \mathcal{N}_{i}\right) \cap J_{i}$ containing $X \backslash \operatorname{leftclip}(X)$, for $X \in \mathcal{N}_{i}^{L}$ and $1 \leqslant i<m$,

- $C_{X}^{R}$ to be the arc-connected component of $\left(\bigcup \mathcal{N}_{i}\right) \cap J_{i}$ containing $X \backslash \operatorname{rightclip}(X)$, for $X \in \mathcal{N}_{i}^{R}$ and $1 \leqslant i<m$,

- $\mathcal{C}$ to be the family of arc-connected components of $\left(\bigcup \mathcal{N}_{i}\right) \cap J_{i}$ for $1 \leqslant i<m$, that is, $\mathcal{C}=\left\{C_{X}^{L}: X \in \mathcal{N}^{L}\right\} \cup\left\{C_{X}^{R}: X \in \mathcal{N}^{R}\right\}$.

For $X \in \mathcal{N}_{i}$ and $1 \leqslant i<m$, the set $X \cap J_{i}$ is arc-connected and thus entirely contained in one member of $\mathcal{C}$. It is clear that $C_{X}^{L} \neq C_{X}^{R}$ for $X \in \mathcal{N}^{L} \cap \mathcal{N}^{R}$. Consider the graph $G$ with vertex set $\mathcal{C}$ and edges connecting $C_{X}^{L}$ and $C_{X}^{R}$ for all $X \in \mathcal{N}^{L} \cap \mathcal{N}^{R}$. We are to show that $G$ is planar.

We construct sets $V \subset E \subset \cup \mathcal{N}$ with the following properties:

(i) $V$ is a finite subset of $\bigcup \mathcal{C}$,

(ii) $E$ is a finite union of arcs with endpoints in $V$, pairwise disjoint outside of $V$,

(iii) $E \cap C$ is arc-connected for every component $C \in \mathcal{C}$,

(iv) every $X \in \mathcal{N}^{L} \cap \mathcal{N}^{R}$ contains an arc in $E$ between $C_{X}^{L}$ and $C_{X}^{R}$.

The construction proceeds by induction on the members of $\mathcal{N}$. We start with $V$ containing one (arbitrary) point in each member of $\mathcal{C}$, and with $E=V$. They clearly satisfy (i)-(iii). Then, for each $X \in \mathcal{N}^{L} \cap \mathcal{N}^{R}$, we enlarge $V$ and $E$ to satisfy the condition (iv) for $X$, as follows. Since $C_{X}^{L} \cup X \cup C_{X}^{R}$ is arc-connected, there is an arc $A \subset C_{X}^{L} \cup X \cup C_{X}^{R}$ between $E \cap C_{X}^{L}$ and $E \cap C_{X}^{R}$. We can furthermore assume that $A \cap E \cap C_{X}^{L}=\left\{v^{L}\right\}$ and $A \cap E \cap C_{X}^{R}=\left\{v^{R}\right\}$. This implies that $A \cap E=\left\{v^{L}, v^{R}\right\}$, as $X \backslash\left(C_{X}^{L} \cup C_{X}^{R}\right)$ is disjoint from any member of $\mathcal{N} \backslash\{X\}$. We add $v^{L}$ and $v^{R}$ to $V$ and $A$ to $E$. After processing all $X \in \mathcal{N}^{L} \cap \mathcal{N}^{R}$, the resulting sets $V$ and $E$ satisfy (i)-(iv).

We have thus obtained a planar representation of a graph $H$ with vertex set $V$ and edge set consisting of maximal arcs in $E$ internally disjoint from $V$. It follows from 
(iii) that the subgraph of $H$ induced on $V \cap C$ is connected for every $C \in \mathcal{C}$. Consider the graph obtained from $H$ by contracting $V \cap C$ for every $C \in \mathcal{C}$. Its vertices represent members of $\mathcal{C}$, and by (iv), its edges connect the vertices representing $C_{X}^{L}$ and $C_{X}^{R}$ for all $X \in \mathcal{N}^{L} \cap \mathcal{N}^{R}$. Hence this graph is isomorphic to $G$. This shows that $G$ is planar, as a contraction of a planar graph is planar.

Since $G$ is planar, there is a proper coloring $\phi$ of the vertices of $G$ with four colors $\{1,2,3,4\}$. Choose a coloring $\psi: \mathcal{N} \rightarrow\{1,2,3,4\}$ so that

- if $X \in \mathcal{N}^{L}$, then $\psi(X)=\phi\left(C_{X}^{L}\right)$,

- if $X \in \mathcal{N}^{R}$, then $\psi(X) \neq \phi\left(C_{X}^{R}\right)$.

Such a coloring exists, because $\phi\left(C_{X}^{L}\right) \neq \phi\left(C_{X}^{R}\right)$ for any $X \in \mathcal{N}^{L} \cap \mathcal{N}^{R}$. To see that $\psi$ is a proper coloring of $\mathcal{N}$, consider some $X, Y \in \mathcal{N}$ such that $X \prec Y$ and $X \cap Y \neq \varnothing$. Since $\phi^{L}(X)=\phi^{L}(Y)$ and $\phi^{R}(X)=\phi^{R}(Y)$ we have $\operatorname{leftclip}(X) \cap \operatorname{leftclip}(Y)=\emptyset$ and $\operatorname{rightclip}(X) \cap \operatorname{rightclip}(Y)=\emptyset$. Therefore,

$$
(X \backslash \operatorname{rightclip}(X)) \cap(Y \backslash \operatorname{leftclip}(Y))=X \cap Y \neq \emptyset .
$$

In particular, we have $X \in \mathcal{N}^{R}$ and $Y \in \mathcal{N}^{L}$. The set $X \cap Y$ is arc-connected, so $C_{X}^{R}=C_{Y}^{L}$. This yields $\psi(X) \neq \phi\left(C_{X}^{R}\right)=\phi\left(C_{Y}^{L}\right)=\psi(Y)$.

Acknowledgments M. Lasoń, P. Micek, and A. Pawlik were supported and B. Walczak was partially supported by Ministry of Science and Higher Education of Poland Grant 884/N-ESF-EuroGIGA/10/2011/0 within ESF EuroGIGA project GraDR. B. Walczak was partially supported by Swiss National Science Foundation Grant 200020-144531.

Open Access This article is distributed under the terms of the Creative Commons Attribution License which permits any use, distribution, and reproduction in any medium, provided the original author(s) and the source are credited.

\section{References}

1. Asplund, E., Grünbaum, B.: On a colouring problem. Math. Scand. 8, 181-188 (1960)

2. Burling, J.P.: On coloring problems of families of prototypes. $\mathrm{PhD}$ thesis, University of Colorado, Boulder (1965)

3. Fox, J., Pach, J.: A separator theorem for string graphs and its applications. Comb. Probab. Comput. 19(3), 371-390 (2010)

4. Fox, J., Pach, J.: Coloring $K_{k}$-free intersection graphs of geometric objects in the plane. Eur. J. Comb. 33(5), 853-866 (2012)

5. Gyárfás, A.: On the chromatic number of multiple interval graphs and overlap graphs. Discrete Math. 55(2), 161-166 (1985)

6. Gyárfás, A.: Corrigendum: on the chromatic number of multiple interval graphs and overlap graphs. Discrete Math. 62(3), 333 (1986)

7. Kim, S.-J., Kostochka, A., Nakprasit, K.: On the chromatic number of intersection graphs of convex sets in the plane. Electron. J. Comb. 11(1), R52 (2004)

8. Kostochka, A., Kratochvíl, J.: Covering and coloring polygon-circle graphs. Discrete Math. 163(1-3), 299-305 (1997)

9. McGuinness, S.: On bounding the chromatic number of L-graphs. Discrete Math. 154(1-3), 179-187 (1996)

10. McGuinness, S.: Colouring arcwise connected sets in the plane I. Graphs Comb. 16(4), 429-439 (2000)

11. Mycielski, J.: Sur le coloriage des graphes. Colloq. Math. 3, 161-162 (1955) 
12. Pawlik, A., Kozik, J., Krawczyk, T., Lasoń, M., Micek, P., Trotter, W.T., Walczak, B.: Triangle-free geometric intersection graphs with large chromatic number. Discrete Comput. Geom. 50(3), 714-726 (2013)

13. Pawlik, A., Kozik, J., Krawczyk, T., Lason, M., Micek, P., Trotter, W.T., Walczak, B.: Triangle-free intersection graphs of line segments with large chromatic number. J. Comb. Theory Ser. B 105, 6-10 (2014)

14. Rok, A., Walczak, B.: Outerstring graphs are $\chi$-bounded. In: Cheng, S.W., Devillers, O. (eds.) 30th Annual Symposium on Computational Geometry (SoCG 2014), pp 136-143. ACM, New York (2014)

15. Suk, A.: Coloring intersection graphs of $x$-monotone curves in the plane. Combinatorica (in press). doi:10.1007/s00493-014-2942-5

16. Zykov, A.A.: On some properties of linear complexes. Mat. Sb. (N.S.) 24(66)(2), 163-188 (1949) (in Russian) 